\title{
Numerical Methods of the Decision Differential the Equations for Continuous Models Of Economy
}

Vorontsova V.L. a

Gorskaya T.Uy. b

ab Kazan Federal University, Institute of Management, Economics and Finance, Kazan, 420008, Russia

\section{Doi:10.5901/mjss.2015.v6n1s3p198}

\section{Abstract}

The review of some models of economy based on application of the ordinary differential equations is provided in article linear and nonlinear, and also the review of approximate methods of their decision. Expediency of use of this or that numerical metods of the solution of the differential equations is shown. For some equations of continuous models of economy in work the approximate method is offered and reasonable. Estimates of convergence of approximate methods are given in the corresponding functional spaces. Results of the numerical solution of these equations in the form of tables and schedules are also received. The comparative analysis of the received results is carried out.

Keywords: differential equations, approximate methods, continuous models of economy.

\section{Introduction}

The review of some models of economy based on application of the ordinary differential equations is provided in article linear and nonlinear, and also the review of approximate methods of their decision. Expediency of use of this or that numerical metol of the solution of the differential equations is shown. For some equations of continuous models of economy in work the approximate method is offered and reasonable. Estimates of convergence of approximate methods are given in the corresponding functional spaces. Results of the numerical solution of these equations in the form of tables and schedules are also received. The comparative analysis of the received results is carried out.

\section{Theory}

It is known $[1,2]$ that some continuous models of economy, for example, investigating economic dynamics, defining evolutions of economic systems are closely connected with the differential equations, with systems of the differential equations of the first order which, in turn, at the decision lead to the equations of the highest orders. So, for example, when studying nature of growth of production in the conditions of the competition, the quantity it is defined as function of time, then, by drawing up the equation of balance of investments into production and research of speed of production, receive the following nonlinear differential equations for finding of quantity of production of $Q(t)$ and determination of nature of its increase:

$$
\begin{aligned}
& Q^{\prime}=\alpha P(Q) Q .(1) \\
& Q^{\prime \prime}=\alpha Q^{\prime}\left(P+\frac{d P}{d Q} Q\right) .
\end{aligned}
$$

At application of dynamic model of Keyes for research of the balance model including the main components of dynamics, account and profitable parts of economy, setting the national income as function of time, we receive the linear differential equation of the first order:

$$
Y^{\prime}=\frac{1-a(t)}{k(t)} Y-\frac{b(t)+E(t)}{k(t)},
$$

where $Y$-dynamics of the national income, $a(t), b(t), k(t), E(t)$ - the set positive functions which are characteristics of functioning and evolution of this state. We will notice also that $a(t)$ is a coefficient of tendency to consumption, the function which isn't reaching unit; $b(t)$ - autonomous (final) consumption; $k(t)$ - norm of acceleration; $E(t)$ - the size defining the public expenditures.

We will consider also neoclassical model of growth in which labor productivity as the function of time determined through $Y=$ by $F(K$ is investigated, $L$ ) - the national income where $F$ - uniform production function of the first order for 
which is fair $F(T K, T L)=T F(K, L)$, To - the volume of capital investments (business assets), $L-$ the volume of expenses of work. We will enter into consideration $\mathrm{k}$ fondovooruzhennost size $=\mathrm{K} / \mathrm{L}$, then labor productivity is expressed by a formula:

$$
f(k)=\frac{F(K, L)}{L}=F(k, 1) \text {. }
$$

The purpose of a task is the description of dynamics of a fondovooruzhennost or its representation as functions from $t$ time. As any model is based on certain prerequisites, we need to make some assumptions and to enter a number of the defining parameters, such as a natural increase of a manpower, an expenditure of investments at increase in business assets at depreciation and other.

As a result we receive the equation of rather unknown function $\mathrm{k}$

$k^{\prime}=l f(k)-(\alpha+\beta) k$,

where function $f(k)$ is defined above.

The received ratio (4) represents the nonlinear differential equation of the first order with the divided variables.

It is possible to give the equations for a market model with the predicted prices as examples of the differential equations of the second order. In simple market models supply and demand is believed usually depending only on the current price of goods. However supply and demand in real situations depend also on a tendency of pricing and rates of the change in price. In models with the continuous and differentiated on time by t functions these characteristics are described according to the first and second derivatives of function of the price of $P(t)$.

We will review a concrete example. Let functions of demand of $D$ and the offer $S$ have the following dependences on the price of $\mathrm{P}$ and its derivatives:

$D(t)=3 P^{\prime \prime}-P^{\prime}-2 P+18$,

$S(t)=4 P^{\prime \prime}+P^{\prime}-3 P+3$.

The dependences accepted here are quite realistic: we will explain it on composed with derivatives of function of the price.

1. Demand "is warmed up" by rate of the change in price: if speed grows $\left(P^{\prime \prime}>0\right)$, the market increases interest in goods, and vice versa. Rapid growth of the price frightens off the buyer therefore composed from the first derivative function of the price enters with a minus sign.

2. The offer in even bigger measure amplifies rate of the change in price therefore the coefficient at $P$ " as $S(t)$ is more, than in $D(t)$. Growth of the price also increases the offer therefore composed, containing $P^{\prime}$, enters expression for $\mathrm{S}(\mathrm{t})$ with a plus.

It is required to establish dependence of the price on time. As the equilibrium condition of the market is characterized by equality of $D=S$, we will equate the right parts of these equations. After reduction of similar members we receive:

$$
P^{\prime \prime}+2 P^{\prime}+5 P=15 \text {. }
$$
function.

The ratio (5) represents the linear non-uniform differential equation of the second order concerning the $P(t)$

Thus, we see that many models of economy lead to the differential equations, both linear, and nonlinear, the first and second orders.

In certain cases it isn't possible to find the exact solution of such equations therefore there is a need to apply numerical methods to finding of the approximate decision of these of the equation. In this regard it would be pertinent to mention approximate methods of the solution of the differential equations.

The choice of this or that numerical method is defined by a variety of reasons. Some of them are features of this class of tasks, requirements imposed to the numerical solution in the field of science and appendices, possibility of computer facilities, and also scientific traditions, qualification of developers.

At the same time, it should be noted also theoretical aspect of research. If Runge-Kutt's method, despite the labor input, possesses considerable accuracy and is widely applied at computer calculation, however, it isn't possible to apply it to theoretical justification of the approximate decision for a wide class of tasks, in a type of the above. Whereas straight lines and projective methods possess this quality.

From a big set of direct methods $[3,4]$ it is possible to allocate group of projective methods, such as Galerkin's method, collocations, subareas, spline methods. They are more convenient for theoretical justification of existence and an assessment of an error of the approximate decision, however, difficult realized in practice.

For the solution of the equations (methods of Ritz, Bubnova - Galerkina) the set of works is devoted to application of straight lines and projective methods $[5,6]$ recently. It is connected with that questions of theoretical researches of these equations and possibility of finding of their optimum decision represent a great interest for modern science. 
Despite the results received for research of the differential equations (see, for example, works [7-9]) now questions of stay and justification of approximate methods of their decision very are particularly acute.

\section{Results}

We will consider a task for the differential equation of a look [10]:

$$
\begin{aligned}
& -\frac{d^{2} u}{d x^{2}}+\frac{d u}{d x}+q u=f(x), x \in(0,1), q>0,(6) \\
& u(0)=u(1)=0,
\end{aligned}
$$

or in an operator form: $A u+B u=f$, where $A u=-d^{2} u / d x^{2}, B u=d u / d x+q u$. As $\mathrm{D}$ (A) we will take a set twice continuously differentiable on $(0,1)$ functions meeting the set regional conditions. The power space generated by the operator $A: H_{A}$-space of the functions belonging to $W_{2}^{1}$, meeting the set boundary conditions. It is possible to take system of functions in qualities of basic functions $\varphi_{i}=\operatorname{sini} \pi x$, full in $H_{A}$ space.

Then the approximate solution of the equation (6) is looked for in a look:

$u_{N}=\sum_{i=1}^{N} a_{i} \operatorname{sini\pi x}$.

Unknown coefficients of $a_{i}$ are defined according to Bubnov-Galerkina's method from the system of the linear algebraic equations which is written down in a matrix look: $L a=f$, где $L=\left\{L_{i j}\right\}, f=\left(f_{1}, \ldots, f_{N}\right)^{\mathrm{T}}$, where

$L_{i j}=\int_{0}^{1}\left(\frac{d \varphi_{i}}{d x} \frac{d \varphi_{j}}{d x}+\frac{d \varphi_{j}}{d x} \varphi_{i}+q \varphi_{i} \varphi_{j}\right) d x, f_{i}=\left(f, \varphi_{i}\right)=\int_{0}^{1} f(x) \varphi_{i}(x) d x$.

Then for the equation (6) the following theorem is fair.

Theorem. Let the operator $A^{-1} B$ be quite continuous in $H_{A}$. Then at enough big $N$ method of Bubnov-Galerkina gives the only approximate solution of $u_{N}$, which meets to the generalized solution $u$ of the equation (6) on norm of $H_{A}$.

The proof of the theorem is kept according to [10]. economy.

Further by way of illustration we will give examples of solutions of the differential equations for some models of

For simplicity we will accept dependence of $P(Q)$ in the equation (2) in the form of the linear $P(Q)=a-b Q$ function. Then the equation (2) will assume an air

$Q^{\prime \prime}=\alpha Q^{\prime}(a-2 b Q)$.

Accepting $a=2 ; a=4 ; b=1$, we will write down $Z$ matrix received as a result of the decision (table 1.).

Table 1. Results of the solution of the equation of speed of release (2):

\begin{tabular}{|r|r|r|r|}
\hline & \multicolumn{1}{|c|}{0} & \multicolumn{1}{|c|}{1} & \multicolumn{1}{c|}{2} \\
\hline 0 & 0 & 0 & 1 \\
\hline 1 & $5 \cdot 10^{-3}$ & $5.101 \cdot 10^{-3}$ & 1.041 \\
\hline 2 & 0.01 & 0.01 & 1.083 \\
\hline 3 & 0.015 & 0.016 & 1.127 \\
\hline 4 & 0.02 & 0.022 & 1.173 \\
\hline 5 & 0.025 & 0.028 & 1.22 \\
\hline 6 & 0.03 & 0.034 & 1.269 \\
\hline 7 & 0.035 & 0.04 & 1.32 \\
\hline 8 & 0.04 & 0.047 & 1.372 \\
\hline 9 & 0.045 & 0.054 & 1.427 \\
\hline 10 & 0.05 & 0.061 & 1.483 \\
\hline 11 & 0.055 & 0.069 & 1.542 \\
\hline 12 & 0.06 & 0.077 & 1.602 \\
\hline 13 & 0.065 & 0.085 & 1.665 \\
\hline 14 & 0.07 & 0.093 & 1.73 \\
\hline 15 & 0.075 & 0.102 & 1.797 \\
\hline
\end{tabular}

Three columns of the table 1 include: the first column - values $t$ in which the decision is looked for; the second column $\mathrm{Q}(\mathrm{t})$; and the third - $\mathrm{Q}^{\prime}(\mathrm{t})$. The decision was received in 300 points in a Mathcad package. At the solution of the differential equation of the second order the vector of entry conditions consists of two elements: values of function and its first derivative in the initial point of an interval.

Further we receive: $Q^{\prime}=0$ at $Q=0$ at and at $Q=a / b ; Q^{\prime \prime}>0$ at $Q<a /(2 b)$ and $Q^{\prime \prime}<0$ at $Q$ $>a /(2 b) ; Q=a /(2 b)=4 / 2=2$ - function graph inflection point $Q=Q(T)$. 1 schedule of this function provided on fig. (one of 
integrated curves of the differential equation (2)) carries the name of a logistic curve.

Fig. 1. Logistic curve.

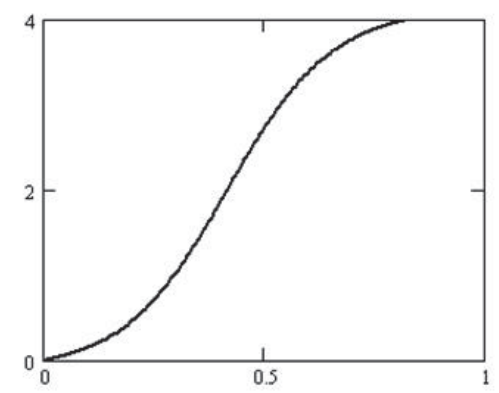

For dynamic model of Keynes in the equation (3) believing key parameters of a task and, b and k constant numbers, we simplify to the linear differential equation of the first order with constant coefficients:

$$
Y^{\prime}=\frac{1-a}{k} Y-\frac{b+E}{k} \text {. }
$$

At $a=0.1 ; b=4 ; E=5 ; k=2$ and the initial moment $Y=2$ matrix of $Z t$ received as a result of the solution of the linear differential equation of the first order with constant coefficients has two columns: the first column contains values $t$ in which the decision is looked for; the second column contains values of the found solution of $Y(t)$ in the corresponding points.

Table 2. Results of the solution of the equation of change of the national income (7):

\begin{tabular}{|c|r|r|}
\hline & \multicolumn{1}{|c|}{0} & \multicolumn{1}{|c|}{1} \\
\hline 0 & 0 & 2 \\
\hline 1 & 0.033 & 1.879 \\
\hline 2 & 0.067 & 1.756 \\
\hline 3 & 0.1 & 1.632 \\
\hline 4 & 0.133 & 1.505 \\
\hline 5 & 0.157 & 1.377 \\
\hline 5 & 0.2 & 1.247 \\
\hline 7 & 0.233 & 1.114 \\
\hline 8 & 0.267 & 0.98 \\
\hline 9 & 0.3 & 0.844 \\
\hline 10 & 0.333 & 0.705 \\
\hline 11 & 0.367 & 0.565 \\
\hline 12 & 0.4 & 0.422 \\
\hline 13 & 0.433 & 0.278 \\
\hline 14 & 0.467 & 0.131 \\
\hline 15 & 0.5 & -0.019 \\
\hline 15 & 0.533 & -0.17 \\
\hline 17 & 0.567 & -0.324 \\
\hline
\end{tabular}

The decision was received in 300 points in a Mathcad package. At the solution of the differential equation of the first order the vector of entry conditions consists of one element: values of function in the initial point of an interval.

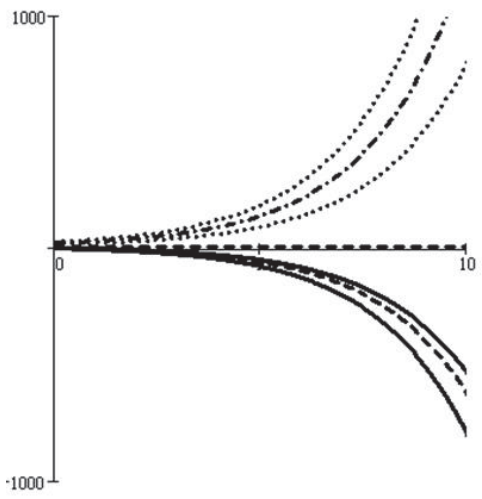

Fig. 2. Family of integrated curves of the equation (7). 
If in an initial time point of $Y_{0}<Y_{p}$, the national income falls at set parameters of $a, b, k$ and $E$ over time. If $Y 0>Y p$, the national income grows in time.

For a market model with the predicted prices, for the equation (5) we will receive the numerical decision in two cases.

We will consider a case 1 . Let at the initial moment of $P(0)=4$ and $P^{\prime}(0)=1$, then Zw matrix received as a result of the solution of the linear non-uniform differential equation of the second order concerning the $P(T)$ function have three columns: the first column contains values $t$ in which the decision is looked for; the second column contains values of the found solution of $P(t)$ in the corresponding points and the third $P^{\prime}(t)$ values.

Decisions were received in 200 points in a Mathcad package.

Table 3. Results of the solution of the equation (7) allowing to establish dependence of the price on time (a case 1):

\begin{tabular}{|c|r|r|r|}
\hline & \multicolumn{1}{|c|}{0} & \multicolumn{1}{c|}{1} & \multicolumn{1}{c|}{2} \\
\hline 0 & 0 & 4 & 1 \\
\hline 1 & 0.05 & 4.041 & 0.662 \\
\hline 2 & 0.1 & 4.067 & 0.348 \\
\hline 3 & 0.15 & 4.077 & 0.059 \\
\hline 4 & 0.2 & 4.073 & -0.202 \\
\hline 5 & 0.25 & 4.057 & -0.437 \\
\hline 6 & 0.3 & 4.03 & -0.643 \\
\hline 7 & 0.35 & 3.993 & -0.823 \\
\hline 8 & 0.4 & 3.948 & -0.976 \\
\hline 9 & 0.45 & 3.896 & -1.102 \\
\hline 10 & 0.5 & 3.838 & -1.203 \\
\hline 11 & 0.55 & 3.776 & -1.281 \\
\hline 12 & 0.6 & 3.71 & -1.336 \\
\hline 13 & 0.65 & 3.643 & -1.369 \\
\hline 14 & 0.7 & 3.574 & -1.384 \\
\hline 15 & 0.75 & 3.505 & -1.38 \\
\hline 16 & 0.8 & 3.436 & -1.361 \\
\hline 17 & 0.85 & 3.369 & -1.327 \\
\hline
\end{tabular}

We will consider a case 2. We will take in the initial moment of $P(0)=4$ and $P^{\prime}(0)=-3$, then Zw1 matrix received as a result of the decision has the appearance given in table 4.

Table 4. Results of the solution of the equation (7) allowing to establish dependence of the price on time (a case 2):

\begin{tabular}{|c|r|r|r|}
\hline & \multicolumn{1}{|c|}{0} & \multicolumn{1}{|c|}{1} & \multicolumn{1}{c|}{2} \\
\hline 0 & 0 & 4 & -3 \\
\hline 1 & 0.05 & 3.852 & -2.934 \\
\hline 2 & 0.1 & 3.707 & -2.84 \\
\hline 3 & 0.15 & 3.568 & -2.721 \\
\hline 4 & 0.2 & 3.435 & -2.581 \\
\hline 5 & 0.25 & 3.31 & -2.424 \\
\hline 6 & 0.3 & 3.193 & -2.253 \\
\hline 7 & 0.35 & 3.085 & -2.071 \\
\hline 8 & 0.4 & 2.986 & -1.882 \\
\hline 9 & 0.45 & 2.897 & -1.689 \\
\hline 10 & 0.5 & 2.817 & -1.494 \\
\hline 11 & 0.55 & 2.748 & -1.299 \\
\hline 12 & 0.6 & 2.687 & -1.108 \\
\hline 13 & 0.65 & 2.637 & -0.922 \\
\hline 14 & 0.7 & 2.595 & -0.743 \\
\hline 15 & 0.75 & 2.562 & -0.571 \\
\hline 16 & 0.8 & 2.538 & -0.41 \\
\hline 17 & 0.85 & 2.521 & -0.259 \\
\hline
\end{tabular}

Results of the solution of the equation (7) allowing to establish dependence of the price on time in two cases are shown on graphics (fig. 3.). 


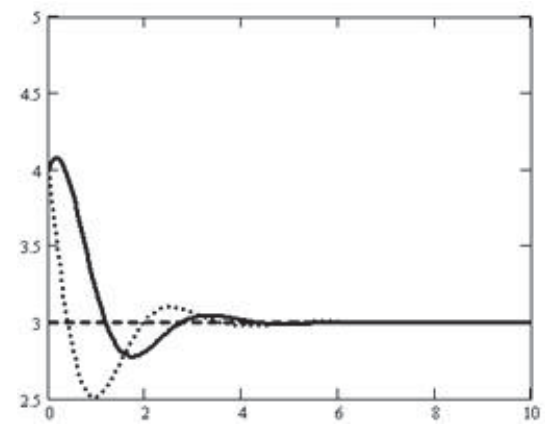

Fig. 3. Family of integrated curves of the equation (7) for cases 1 and 2.

From the schedule it is visible that all prices aspire to the established PSt=3 price with fluctuations about it, and amplitude of these fluctuations fades over time. That will be coordinated with known results from the economic theory [11].

\section{Conclusions}

Investigating various differential equations for continuous models of economy it is necessary to understand that RungeKutt's method of the fourth order is the most common for the ordinary differential equations. The formulation "the most common" is connected with the developed tendency in use of numerical methods. At formulas of an identical order of accuracy the main members of an error on a step often are disproportionate. For one equations one method, and for others - another gives a smaller error. In a similar situation of the recommendation in favor of this or that method have to be based on the "strong-willed decision" made taking into account traditions and practice of use of methods. The concept of practice of computing work is quite uncertain. However, despite such uncertainty, criterion of practice often bears in itself certain positive information which often at this stage of development of science can't be formalized or proved.

\section{References}

Crassus M. S., Chuprynov B. P. Fundamentals of mathematics and its appendix in economic education: Textbook. - M.: Business, 2002. - pp. 180-191.

Ross S. I. Mathematical modeling and management of national economy: Educational grant / SPb.: Publishing house of SPb of GU ITMO, 2006.

Samarsky A. A. Numerical methods / A. A. Samarsky, A. V. Gulin. - M.: Science, 1989.

Semushin, I.V. Algebras / I.V numerical methods. Semushin. - Ulyanovsk: UISTU, 2006.

Gorskaya T.YU., Ojegova A.V. About convergence of a projective method for the equation of a problem of the movement // KGASU News, 2013, 2(24). - pp. 112-126.

Gorskaya T.YU., Zolotonosov Ya.D., Barmin K.E. Metod of a collocation for the equation of a problem of the movement // KGASU News, 2013, No. 4(26). - pp. 175-180.

Vorontsova V. L. About the analysis of behavior of limit cycles with a growth of eccentricity of an orbit and aerodynamic parameter // Messenger of the Moscow Aviation institute № 1, V. 20, 2013. - pp. 255-258.

Goncharova E.N., Vorontsova V. L. Identification of limit cycles at the movement of a ligament of two bodies under the influence of aerodynamics // the SGU Bulletin № 57(4), 2008. - pp. 14-17.

Vorontsova V. L. About research of behavior of limit cycles depending on increase of eccentricity // the SGU Bulletin № 77(1), 2011. pp. 304-306.

Marchuk G. I., Agoshkov V. I. Introduction to projective and net methods. - M.: Science, 1981. - 416 p.

Bases of the econometric analysis of data: Manual / E.G.Zhilyakov., Yu.M.Perlov., E.P.Revtova. Belgorod 2004.

Nagimova, A.M., Safiullina, F.R. (2014). Combination of university training with employment among Kazan' students. Sotsiologicheskie Issledovaniya, (4), pp. 121-124.

Tsertseil, J.S. The way of clusters uprising and development in the region (By the example of the petrochemical cluster in the republic of Tatarstan). Mediterranean Journal of Social Sciences, Volume 5, Issue 18 SPEC. ISSUE, 2014, Pages 125-128

Safina, D., Podgornaya, A. (2014). Mobbing as an organizational phenomenon impeding implementation of changes. Mediterranean Journal of Social Sciences, 5 (18 SPEC. ISSUE), pp. 187-192. 